\title{
Cosmic ray small-scale anisotropies in quasi-linear theory
}

\author{
Philipp Mertsch* \\ Institute for Theoretical Particle Physics and Cosmology (TTK), RWTH Aachen University, \\ 52056 Aachen, Germany \\ E-mail: pmertsch@physik.rwth-aachen.de \\ Markus Ahlers \\ Niels Bohr International Academy, Niels Bohr Institute, Blegdamsvej 17, 2100 Copenhagen, \\ Denmark \\ E-mail: markus.ahlers@nbi.ku.dk
}

\begin{abstract}
The distribution of arrival directions of cosmic rays is remarkably isotropic, which is a consequence of their repeated scattering in magnetic fields. Yet, high-statistics observatories like IceCube and HAWC have revealed the presence of small-scale structures at levels of 1 part in 10,000 at hundreds of $\mathrm{TeV}$, which are not expected in typical diffusion models of cosmic rays. We follow up on the suggestion that these small-scale anisotropies are a result of cosmic ray streaming in a particular realisation of the turbulent magnetic field within a few scattering lengths in our local Galactic neighbourhood. So far, this hypothesis has been investigated mostly numerically, by tracking test particles through turbulent magnetic fields. For the first time, we present an analytical computation that through a perturbative approach allows predicting the angular power spectrum of cosmic ray arrival directions for a given model of turbulence. We illustrate this method for a simple, isotropic turbulence model and we find remarkable agreement with the results of numerical studies.
\end{abstract}

36th International Cosmic Ray Conference -ICRC2019-

July 24th - August 1st, 2019

Madison, WI, U.S.A.

\footnotetext{
* Speaker.
} 


\section{Introduction}

The arrival directions of cosmic rays (CRs) are highly isotropic. Usually, this is explained as a consequence of pitch-angle scattering between CRs and turbulent magnetic fields. If the largescale distribution of $\mathrm{CR}$ sources results in a spatial gradient, quasi-linear theory $[1,2,3,4,5]$ predicts a small dipole anisotropy. Yet, observations show fluctuations on smaller scales, down to $10^{\circ}$ degrees. These small-scale anisotropies are conveniently quantified by the angular-power spectrum, defined as

$$
C_{\ell}(t) \equiv \frac{1}{4 \pi} \int \mathrm{d} \widehat{\boldsymbol{p}}_{A} \int \mathrm{d} \widehat{\boldsymbol{p}}_{B} P_{\ell}\left(\widehat{\boldsymbol{p}}_{A} \cdot \widehat{\boldsymbol{p}}_{B}\right) f_{A} f_{B}
$$

where we use the abbreviation $\widehat{\boldsymbol{p}}=\boldsymbol{p} /|\boldsymbol{p}|$ and $f_{A}=f\left(0, \boldsymbol{r}_{0}, \boldsymbol{p}_{A}\right)$, etc. Small-scale anisotropies are

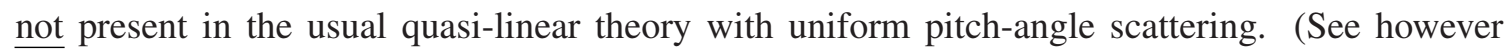
Ref. [6]). For a recent review on observations and interpretations of the small-scale anisotropies see Ref. [7]

One of the arguably most attractive explanations of the small-scale anisotropies is that they are due to magnetic turbulence itself $[8,9,10]$. Standard quasi-linear theory only predicts the ensemble-averaged phase-space density $\langle f\rangle$ and we can therefore only predict the angular power spectrum $C_{\ell}^{\text {std }}$ obtained from Eq. (1.1) through $f_{A} f_{B} \rightarrow\left\langle f_{A}\right\rangle\left\langle f_{B}\right\rangle$. Under the commonly used assumptions $C_{\ell}^{\text {std }} \sim 0$ for $\ell \geq 2$. (See again Ref. [6] for modifications to this simple picture.) However, it is easy to see that in the ensemble-average the angular power spectrum $\left\langle C_{\ell}\right\rangle$ can have small-scale power, i.e. $\left\langle C_{\ell}\right\rangle>C_{\ell}^{\text {std }}$, if $\left\langle f_{A} f_{B}\right\rangle>\left\langle f_{A}\right\rangle\left\langle f_{B}\right\rangle$. In other words, if there are correlations between the fluxes of CRs arriving under an angle $\theta \equiv \arccos \left(\widehat{\boldsymbol{p}}_{A} \cdot \widehat{\boldsymbol{p}}_{B}\right) \sim \pi / \ell$ (with $\ell$ the orbital quantum number corresponding to this angle $\theta$ ) then the average angular power spectrum $\left\langle C_{\ell}\right\rangle$, computed from the ensemble average of the product of phase-space densities, will be larger than the standard angular power spectrum $C_{\ell}^{\text {std }}$, computed from the product of ensemble-averaged phase-space densities. Therefore, correlations lead to small-scale anisotropies.

These correlations are to be expected if particles propagate through a turbulent magnetic field: Particles arriving under an angle $\theta$ will have experienced similar fields for a certain amount of time before observation. It can be motivated [9] that this time is of the order $\tau_{\mathrm{sc}} /(\ell(\ell+1))$ where $\tau_{\mathrm{sc}}$ is the scattering time. It is therefore ultimately the spatial correlations of the turbulent magnetic field that are reflected in the angular correlations of CR arrival directions.

In the following we will predict the angular power spectrum Eq. (1.1) in an extended quasilinear theory, taking into account the angular correlation between phase-space densities. We will consider the case with an isotropic turbulence tensor and without regular magnetic field. In this configuration, the unperturbed trajectories are straight lines, thus particles are propagating ballistically.

\section{Single-Particle Propagator}

In the following, we will make use of a diagrammatic formalism for solving stochastic differential equations, as used for instance in propagation of waves through random media. Here, we briefly review this formalism to fix our notation. We refer the interested reader to Refs. [11, 12] for details. 
The problem of propagation of (relativistic) charged particles through a static regular and turbulent magnetic field $\boldsymbol{B}_{0}$ and $\boldsymbol{\delta} \boldsymbol{B}(\boldsymbol{r})$ can be formulated using Liouville's equation for the phasespace density $f=f(\boldsymbol{r}, \boldsymbol{p}, t)$,

$$
\partial_{t} f+c \widehat{\boldsymbol{p}} \cdot \boldsymbol{\nabla} f+\mathscr{L}_{0} f=-\delta \mathscr{L}(t) f
$$

with the deterministic and stochastic Liouville operators

$$
\mathscr{L}_{0}=-i \boldsymbol{\Omega} \cdot \boldsymbol{L} \quad \text { and } \quad \delta \mathscr{L}=-i \boldsymbol{\omega}(\boldsymbol{r}) \cdot \boldsymbol{L},
$$

where $\boldsymbol{\Omega}=q \boldsymbol{B}_{0} / p_{0}$ and $\boldsymbol{\omega}(\boldsymbol{r})=q \boldsymbol{\delta} \boldsymbol{B}(\boldsymbol{r}) / p_{0}$ are the (relativistic) gyrovectors of the regular and turbulent field, respectively, and $L_{i} \equiv-i \varepsilon_{i j k} p_{j} \partial_{p_{k}}$ are angular momentum operators.

In the following, we will assume that the spatial dependence of the phase-space density can be approximated by the first two terms of a Taylor expansion,

$$
f(\boldsymbol{r}, \boldsymbol{p}, t) \simeq f_{\odot}(\boldsymbol{p}, t)+\left(\boldsymbol{r}-\boldsymbol{r}_{\odot}\right) \cdot \boldsymbol{G} .
$$

where $f_{\odot}(\boldsymbol{p}, t) \equiv f\left(\boldsymbol{r}_{\odot}, \boldsymbol{p}, t\right)$ denotes the local phase-space density and $\boldsymbol{G}$ a constant gradient vector. With this ansatz, the Liouville equation 2.1 evaluates to

$$
\partial_{t} f_{\odot}+\mathscr{L}_{0} f_{\odot}+\delta \mathscr{L} f_{\odot} \simeq c \widehat{\boldsymbol{p}} \cdot \boldsymbol{G} .
$$

This can be formally solved as

$$
f_{\odot}(\boldsymbol{p}, t) \simeq U_{t_{0}, t} f_{\odot}\left(\boldsymbol{p}, t_{0}\right)+\int_{t_{0}}^{t} \mathrm{~d} t^{\prime} U_{t^{\prime}, t} c \widehat{\boldsymbol{p}} \cdot \boldsymbol{G}=U_{t_{0}, t} f_{\odot}\left(\boldsymbol{p}, t_{0}\right)+\Delta \boldsymbol{r}\left(t_{0}\right) \cdot \boldsymbol{G},
$$

with $\Delta \boldsymbol{r}\left(t_{0}\right) \equiv \boldsymbol{r}\left(t_{0}\right)-\boldsymbol{r}_{\odot}$ and the aid of the time-evolution operator (also called propagator), written using the time-ordered ("latest-to-left") exponential,

$$
U_{t, t_{0}}=\mathscr{T} \exp \left[-\int_{t_{0}}^{t} \mathrm{~d} t^{\prime}\left(\mathscr{L}_{0}+\delta \mathscr{L}\left(t^{\prime}\right)\right)\right]=U_{t, t_{0}}^{(0)} \mathscr{T} \exp \left[-\int_{t_{0}}^{t} \mathrm{~d} t^{\prime}\left(U_{t^{\prime}, t_{0}}^{(0)}\right)^{-1} \delta \mathscr{L}\left(t^{\prime}\right) U_{t^{\prime}, t_{0}}^{(0)}\right] .
$$

Here, $U_{t, t_{0}}^{(0)}$ denotes the free propagator,

$$
U_{t, t_{0}}^{(0)}=\exp \left[-\left(t-t_{0}\right) \mathscr{L}_{0}\right] .
$$

Note that $U_{t, t_{0}}^{-1}=U_{t_{0}, t}$, which we use in Eq. (2.5).

What complicates the solution of Eq. (2.1) is the stochastic nature of $\delta \mathscr{L}\left(t^{\prime}\right)$. One can only hope to predict moments of the propagator, its first moment being the expectation value. In the Gaussian limit, the expectation value of the propagator, $\left\langle U_{t, t_{0}}\right\rangle$ contains only two-point functions of $\delta \mathscr{L}\left(t^{\prime}\right)$,

$$
\left\langle\delta \mathscr{L}\left(t_{n}\right) \delta \mathscr{L}\left(t_{n-1}\right) \ldots \delta \mathscr{L}\left(t_{1}\right)\right\rangle=\left\langle\delta \mathscr{L}\left(t_{n}\right) \delta \mathscr{L}\left(t_{n-1}\right)\right\rangle \ldots\left\langle\delta \mathscr{L}\left(t_{1}\right) \delta \mathscr{L}\left(t_{0}\right)\right\rangle+\text { permut. }
$$

for even $n$ and vanishes identically for odd $n$. The expansion of Eq. (2.6) becomes algebraically complex very quickly. It can be diagrammatically written in a more economic form,

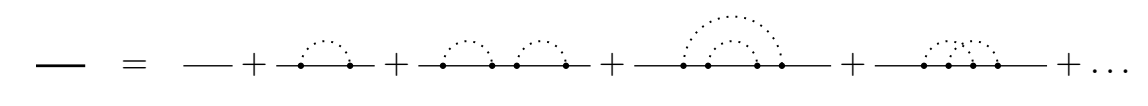

Here, solid lines represent free single-particle propagators $U_{t, t^{\prime}}^{(0)}$, dots correspond to insertions of $\delta \mathscr{L}\left(t^{\prime}\right)$ and dotted lines connecting such dots represent the expectation value of the two $\delta \mathscr{L}$ 's that it connects. All intermediate time variables are integrated over. 


\section{Pair Propagator}

The small-scale anisotropies are a consequence of the fact that the trajectories of a pair of CRs are correlated for a (finite) amount of time before observation. Therefore, we need to consider the ensemble average of products of phase-space densities when computing the angular power spectrum. Note that in standard quasi-linear theory, we compute the ensemble average of single phase-space densities and are therefore missing the correlations between pairs of CR particles.

In the following, we will use the abbreviations $f_{A}(t) \equiv f_{\odot}\left(\boldsymbol{p}_{A}, t\right)$, etc. From eq. (2.5), we find for the ensemble average of the product of phase-space densities,

$$
\begin{aligned}
\left\langle f_{A}(t) f_{B}^{*}(t)\right\rangle \simeq & \left.\left\langle U_{t_{0}, t}^{A} U_{t_{0}, t}^{B *}\right\rangle\left\langle f_{A}\left(t_{0}\right) f_{B}^{*}\left(t_{0}\right)\right\rangle+\left\langle\left(\Delta \boldsymbol{r}_{A}\left(t_{0}\right) \cdot \boldsymbol{G}\right) U_{t_{0}, t}^{B *}\right\rangle f_{B}^{*}\left(t_{0}\right)\right\rangle \\
& +\left\langle\left(\Delta \boldsymbol{r}_{B}\left(t_{0}\right) \cdot \boldsymbol{G}\right) U_{t_{0}, t}^{A}\right\rangle\left\langle f_{A}\left(t_{0}\right)\right\rangle+\left\langle\left(\Delta \boldsymbol{r}_{A}\left(t_{0}\right) \cdot \boldsymbol{G}\right)\left(\Delta \boldsymbol{r}_{B}\left(t_{0}\right) \cdot \boldsymbol{G}\right)\right\rangle
\end{aligned}
$$

In our previous analysis [10] we identified the last term on the right of Eq. (3.1) as the term that determines the asymptotic behavior of the power-spectrum for large look-back times, $t-t_{0} \gg v^{-1}$. $v$ can be interpreted as a scattering time and contains integrals over the two-point functions of the turbulent field $\omega(\boldsymbol{r})$. Here, we aim to establish a differential equation in quasi-linear theory based on Eq. (3.1). Note that we have assumed that correlations between the propagators and the initial state $f_{\odot}\left(\boldsymbol{p}, t_{0}\right)$ can be ignored.

By virtue of the Bethe-Salpeter equation [13], the double propagator can be expanded into a perturbative series. This series has a diagrammatic representation, somewhat similar to Feynman diagrams employed in quantum field theory,

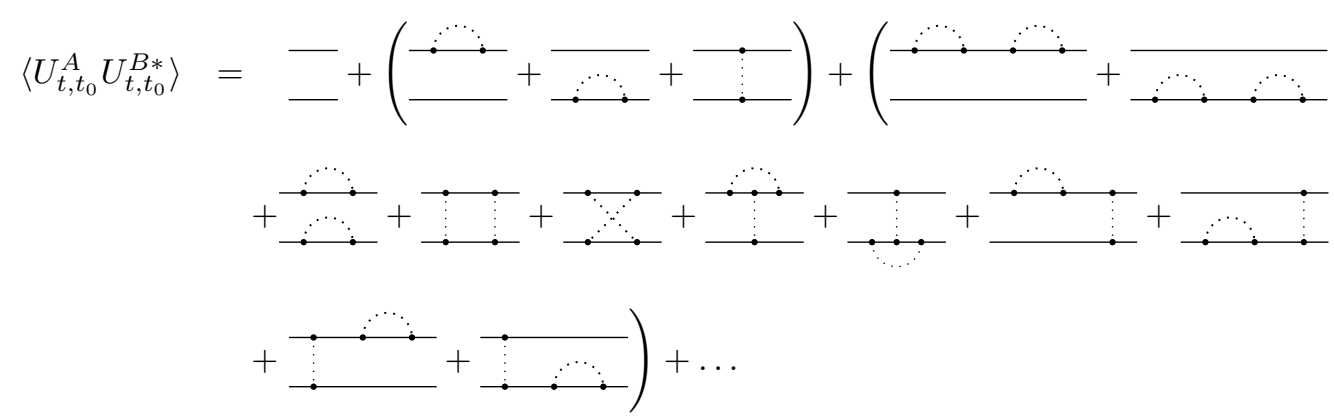

If the dashed lines are connecting the Liouville operators $\delta \mathscr{L}$ of two different particles $\mathrm{A}$ and $\mathrm{B}$, then this can be considered an interaction between particles $\mathrm{A}$ and $\mathrm{B}$ mediated by the correlation structure of the turbulent magnetic field. It is the repeated action of these "interactions" that is inducing the correlations between particles A and B.

While eq. (3.1) allows computing the APS anytime after preparing the initial state, $f\left(\widehat{\boldsymbol{p}}, t_{0}\right)$, evaluating or even resumming all diagrams of Eq. (3.2) in all generality seems challenging at the very least. Instead, we seek to approximate the identity (3.1) by the stationary solution of a differential equation with respect to a small step in look-back time $\Delta T \equiv t-t_{0}$,

$$
\begin{aligned}
& \frac{1-\left\langle U_{t_{0}, t}^{A} U_{t_{0}, t}^{B *}\right\rangle}{\Delta T}\left\langle f_{A}\left(t_{0}\right) f_{B}^{*}\left(t_{0}\right)\right\rangle \\
& \simeq\left(\phi_{\odot}-3 \widehat{\boldsymbol{p}}_{A} \cdot \mathbf{K} \cdot \boldsymbol{G}\right)\left(\frac{\Delta \boldsymbol{r}_{B}}{\Delta T} \cdot \boldsymbol{G}\right)+\left(\frac{\Delta \boldsymbol{r}_{A}}{\Delta T} \cdot \boldsymbol{G}\right)\left(\phi_{\odot}-3 \widehat{\boldsymbol{p}}_{B} \cdot \mathbf{K} \cdot \boldsymbol{G}\right)+\mathscr{O}(\Delta T),
\end{aligned}
$$


where we applied the quasi-stationary solution of the diffusion equation $\left\langle f_{A}\left(t_{0}\right)\right\rangle \simeq \boldsymbol{\phi}_{\odot}-3 \widehat{\boldsymbol{p}}_{A} \cdot \mathbf{K} \cdot \boldsymbol{G}$. In this limit $\Delta T \rightarrow 0$, we can approximate $\Delta \boldsymbol{r} / \Delta T \simeq-\widehat{\boldsymbol{p}}$. This allows writing down an ordinary differential equation for the angular power spectrum $C_{\ell}$,

$$
A_{\ell \ell_{0}} C_{\ell_{0}}(t) \simeq \frac{8 \pi}{9} K|\boldsymbol{G}|^{2} \delta_{\ell 1},
$$

where we assume isotropic diffusion $K_{i j}=K \delta_{i j}$ and define the transition matrix

$$
A_{\ell \ell_{0}}\left(t_{0}\right)=\lim _{t_{0} \rightarrow t} \frac{\delta_{\ell \ell_{0}}-M_{\ell \ell_{0}}\left(t, t_{0}\right)}{t-t_{0}}
$$

and where

$$
M_{\ell \ell_{0}}\left(t, t_{0}\right)=\frac{1}{4 \pi} \int \mathrm{d} \widehat{\boldsymbol{p}}_{A} \int \mathrm{d} \widehat{\boldsymbol{p}}_{B} P_{\ell}\left(\widehat{\boldsymbol{p}}_{A} \cdot \widehat{\boldsymbol{p}}_{B}\right)\left\langle U_{t, t_{0}}^{A} U_{t, t_{0}}^{B *}\right\rangle \frac{2 \ell_{0}+1}{4 \pi} P_{\ell_{0}}\left(\widehat{\boldsymbol{p}}_{A} \cdot \widehat{\boldsymbol{p}}_{B}\right) .
$$

Once we have computed $M_{\ell \ell_{0}}\left(t, t_{0}\right)$, it is easy to find the steady-state angular power spectrum $C^{\text {stdy }}$ by solving

$$
A_{\ell \ell_{0}} C_{\ell_{0}}^{\text {stdy }}(t)=\frac{8 \pi}{9} K|\boldsymbol{G}|^{2} \delta_{\ell 1}
$$

In evaluating $\left\langle U_{t, t_{0}}^{A} U_{t, t_{0}}^{B *}\right\rangle$, we confine ourselves to considering the leading and next-to-leading order terms, that is the first line of Eq. (3.2). We label the contributions of those diagrams to the double propagator as follows,

$$
\begin{aligned}
& \left(\left\langle U_{t, t_{0}}^{A} U_{t, t_{0}}^{B *}\right\rangle\right)_{0}=-, \quad\left(\left\langle U_{t, t_{0}}^{A} U_{t, t_{0}}^{B *}\right\rangle\right)_{1 a}=\stackrel{? \cdot}{\square}, \\
& \left(\left\langle U_{t, t_{0}}^{A} U_{t, t_{0}}^{B *}\right\rangle\right)_{1 b}=\stackrel{\cdots}{\because \cdots}, \quad\left(\left\langle U_{t, t_{0}}^{A} U_{t, t_{0}}^{B *}\right\rangle\right)_{1 c}=\square
\end{aligned} .
$$

Evaluating Eq.(3.6) for all diagrams/contributions of Eqs. (3.8) and (3.9) we find (see [14] for details),

$$
\begin{aligned}
M_{\ell \ell_{0}}^{0} & =\delta_{\ell \ell_{0}} . \\
M_{\ell \ell_{0}}^{(1 a)} & =-\frac{8 \pi}{3} \delta_{\ell \ell_{0}}\left(\Lambda_{0}(\Delta T)-\frac{1}{2} \Lambda_{2}(\Delta T)\right) \ell(\ell+1) . \\
M_{\ell \ell_{0}}^{(1 c, 0)} & =\frac{8 \pi}{3} \sum_{\ell_{A}}\left(2 \ell_{A}+1\right) \kappa_{\ell_{A} \ell_{A}}\left(t-t_{0}\right)\left(\begin{array}{ccc}
\ell & \ell_{A} & \ell_{0} \\
0 & 0 & 0
\end{array}\right)^{2}\left(2 \ell_{0}+1\right) \ell_{0}\left(\ell_{0}+1\right), \\
M_{\ell, \ell_{0}}^{(1 c, 2)} & =\frac{4 \pi}{3} \frac{\ell_{0}\left(\ell_{0}+1\right)\left(2 \ell_{0}+1\right)}{\left(\begin{array}{ccc}
\ell_{0} & 2 & \ell_{0} \\
0 & 0 & 0
\end{array}\right)}(-1)^{\ell_{0}} \sum_{\ell_{A}, \ell_{B}} \imath^{\ell_{A}+\ell_{B}} \kappa_{\ell_{A} \ell_{B}}\left(t-t_{0}\right)\left(2 \ell_{A}+1\right)\left(2 \ell_{B}+1\right) \\
& \times\left\{\begin{array}{ccc}
2 & \ell_{0} & \ell_{0} \\
\ell & \ell_{A} & \ell_{B}
\end{array}\right\}\left(\begin{array}{ccc}
2 & \ell_{A} & \ell_{B} \\
0 & 0 & 0
\end{array}\right)\left(\begin{array}{ccc}
\ell & \ell_{A} & \ell_{0} \\
0 & 0 & 0
\end{array}\right)\left(\begin{array}{ccc}
\ell & \ell_{B} & \ell_{0} \\
0 & 0 & 0
\end{array}\right) .
\end{aligned}
$$

Here, we have defined

$$
\begin{aligned}
\Lambda_{\ell_{A}}(\Delta T) & \equiv \int_{0}^{\Delta T} \mathrm{~d} T \int_{0}^{T} \mathrm{~d} \tau \int \mathrm{d} k g(k) j_{\ell_{A}}(k \tau), \\
\kappa_{\ell_{A} \ell_{B}}(\Delta T) & \equiv \int_{k_{0}}^{k_{1}} \mathrm{~d} k \frac{g(k)}{k^{2}} \int_{0}^{\Delta T} \mathrm{~d} \tau_{A} j_{\ell_{A}}\left(k \tau_{A}\right) \int_{0}^{\Delta T} \mathrm{~d} \tau_{B} j_{\ell_{B}}\left(k \tau_{B}\right),
\end{aligned}
$$


and

$$
\kappa_{\ell_{A} \ell_{B}}(\Delta T) \equiv \int_{k_{0}}^{k_{1}} \mathrm{~d} k \frac{g(k)}{k^{2}} h_{\ell_{A}}(k, \Delta T) h_{\ell_{B}}(k, \Delta T),
$$

and $(\cdots)$ denotes the Wigner $3 j$-symbol and $\{\cdots\}$ the Wigner $6 j$-symbol.

Eventually, we compute $M_{\ell \ell_{0}}=M_{\ell \ell_{0}}^{(0)}+2 M_{\ell \ell_{0}}^{(1 a)}+M_{\ell \ell_{0}}^{(1 c, 0)}+M_{\ell \ell_{0}}^{(1 c, 2)}$ (see Eqs. (3.10), (3.11), (3.12) and (3.13)), determine $A_{\ell \ell_{0}}$ from Eq. (3.5) and find the steady-state angular power spectrum from eq. (3.7).

\section{Validation}

In order to validate the results of our analytical computation we now compare to numerical results following the method in Ref. [10]. The power spectrum can be derived from the last term of Eq. (3.1) in the limit $\Delta T \rightarrow \infty$. We have back-tracked test-particles in isotropic turbulent magnetic fields with band-limited white-noise spectrum. We have not assumed any regular component. Specifically, we have chosen $k_{0} r_{g}=10^{-3}$ and $k_{1} r_{g}=10^{2}$, with $r_{g}$ the particles gyroradius in the RMS turbulent field. This guarantees that there is a broad enough range of wavenumbers to be in resonance with $\left(r_{g} k_{\text {res }} \approx 1, k_{\text {res }}\right.$ being the resonant wave number) while satisfying the requirement $k_{1} / k_{0} \gg 1$. The numerical backtracking results in a set of trajectories that converge at $\boldsymbol{r}_{\odot}$. Thanks to Liouville's theorem, we can use this to compute the angular distribution at position $\boldsymbol{r}_{\odot}$ and time $t$ by assuming a certain phase-space density at time $t_{0}$. To make the connection with our analytical approach, we adopt the same gradient dependence as in Eq. 2.3. For each of 100 random realisations of the turbulent magnetic field, we compute the angular power spectrum from the phase-space density at position $\boldsymbol{r}_{\odot}$ and time $t$ and finally compute the ensemble averaged angular power spectrum.

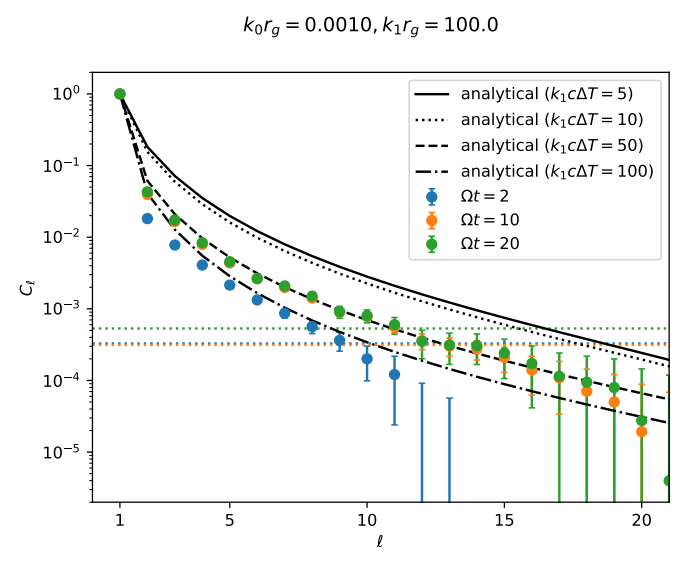

Figure 1: Validation of the analytical method by comparison with numerical result. The data points show the angular power spectrum determined in testparticle simulations for three different backtracking times $t-t_{0}$ after subtraction of the estimated shot noise. The shot noise levels due to the finite number of trajectories is indicated by the horizontal dashed lines. For comparison, the lines show the results of our analytical approach with different values of the free parameter $k_{1} c \Delta T$. Fixing this free parameter to $k_{1} c \Delta T \approx 50$ results in excellent agreement with the test-particle simulations. 
In Fig. 1, we show this ensemble averaged angular power spectrum for three different backtracking times $\left(t-t_{0}\right)$. It can be seen that the angular power spectrum converges to an asymptotic form $\Omega t \gtrsim 10$ where $\Omega$ is the gyro frequency in the RMS turbulent field. (See also Ref. [10].) Comparing with the analytical results allows fixing the free parameter, $k_{1} c \Delta T$, for which we otherwise only have the constraint $k_{1} c \Delta T \gg 1$. It appears that $k_{1} c \Delta T \approx 50$ gives excellent agreement between analytical and numerical results.

\section{Results}
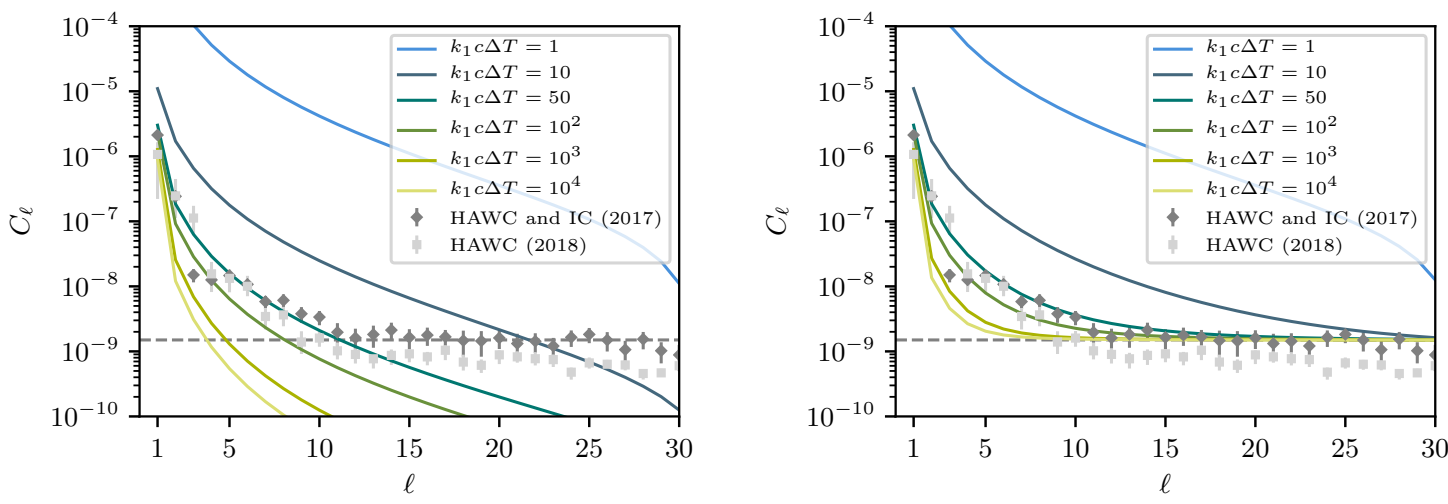

Figure 2: The angular power spectrum computed in quasi-linear theory for different values of $k_{1} c \Delta T$, without (left panel) and with (right panel) adding the noise contribution. For comparison, we also show the observations by HAWC [15] and the IceCube-HAWC combined data [16] with the shot noise level estimated for the latter.

While the band-limited white-noise spectrum serves only as an approximation for the magnetic turbulence in our local environment, it is nevertheless instructive to compare our model predictions to the power spectrum observed by HAWC and IceCube [15, 16]. The solide lines in Fig. 2 show the steady-state angular power spectrum $C^{\text {stdy }}$ derived by numerically solving Eq. (3.7). In the left panel, we have fixed the gradient $K|\boldsymbol{G}|^{2}=10^{-4} c k_{0}$ and show the result for a range of $k_{1} c \Delta T$. It can be seen that with increasing values of $k_{1} c \Delta T$, the normalisation of the angular power spectrum is decreasing and the power spectrum tends to fall off much faster. We compare our model predictions to the angular power spectra inferred from HAWC data [15] and the combined IceCube-HAWC data [16]. Note that we have not accounted for the shot noise necessarily present in the data or for cross talk between multipole moments due to IceCube's limited field of view, see Ref. [7]. In the right panel of Fig. 2, we do account for the effect of shot noise by adding a constant noise power of $\mathscr{N} \simeq 1.5 \times 10^{9}$. This is reproducing the data from the combined analysis of HAWC and IceCube data [16] which is dominated by shot noise above $\ell \gtrsim 10$. Overall, with $\alpha=k_{1} c \Delta T \simeq 50$ we find good agreement with the data, again for $K|\boldsymbol{G}|^{2}=10^{-4} c k_{0}$.

\section{Summary and Conclusion}

We have presented a computation of the angular power spectrum $C_{\ell}$ of cosmic ray anisotropies in quasi-linear theory, based on a perturbative expansion of the time-evolution operator. In particular, we have computed the $C_{\ell}$ 's as the steady-state solution of an ordinary differential equation for 
the time-evolution of the $C_{\ell}$ 's. For the power spectrum of small-scale magnetic turbulence we have assumed band-limited white-noise. We have validated our approach with a numerical simulation of particle trajectories in the same kind of turbulence. Applying our analytical results to data from the HAWC and IceCube experiments we find remarkable agreement.

\section{Acknowledgments}

This was supported by Danmarks Grundforskningsfond under grant no. 1041811001. MA also acknowledges support from VILLUM FONDEN (project no. 18994).

\section{References}

[1] J. R. Jokipii, Cosmic-Ray Propagation. I. Charged Particles in a Random Magnetic Field, Astrophys. J. 146 (Nov., 1966) 480.

[2] C. F. Kennel and F. Engelmann, Velocity Space Diffusion from Weak Plasma Turbulence in a Magnetic Field, Physics of Fluids 9 (Dec., 1966) 2377-2388.

[3] D. E. Hall and P. A. Sturrock, Diffusion, Scattering, and Acceleration of Particles by Stochastic Electromagnetic Fields, Physics of Fluids 10 (Dec., 1967) 2620-2628.

[4] K. Hasselmann and G. Wibberenz, A Note on the Parallel Diffusion Coefficient, Astrophys. J. 162 (Dec., 1970) 1049.

[5] J. R. Jokipii, Fokker-Planck Equations for Charged-Particle Transport in Random Fields., Astrophys. J. 172 (Mar., 1972) 319.

[6] G. Giacinti and J. G. Kirk, Large-Scale Cosmic-Ray Anisotropy as a Probe of Interstellar Turbulence, Astrophys. J. 835 (2017), no. 2 258, [arXiv: 1610 . 0613].

[7] M. Ahlers and P. Mertsch, Origin of Small-Scale Anisotropies in Galactic Cosmic Rays, Prog. Part. Nucl. Phys. 94 (2017) 184-216, [arXiv:1612.0187].

[8] G. Giacinti and G. Sigl, Local Magnetic Turbulence and TeV-PeV Cosmic Ray Anisotropies, Phys. Rev. Lett. 109 (2012) 071101, [arXiv:1111.2536].

[9] M. Ahlers, Anomalous Anisotropies of Cosmic Rays from Turbulent Magnetic Fields, Phys. Rev. Lett. 112 (2014), no. 2 021101, [arXiv: 1310.5712 ].

[10] M. Ahlers and P. Mertsch, Small-Scale Anisotropies of Cosmic Rays from Relative Diffusion, Astrophys. J. 815 (2015), no. 1 L2, [arXiv: 1506.0548 ].

[11] U. Frisch, Wave propagation in random media, in Probabilistic Methods in Applied Mathematics (A. T. Bharucha-Reid, ed.), vol. 1, ch. 2, pp. 75-198. Academic Press, Inc., New York, 1968.

[12] G. Pelletier, Renormalization method and singularities in the theory of Langmuir turbulence, Journal of Plasma Physics 18 (Aug., 1977) 49-76.

[13] E. E. Salpeter and H. A. Bethe, A Relativistic Equation for Bound-State Problems, Physical Review 84 (Dec., 1951) 1232-1242.

[14] P. Mertsch and M. Ahlers, "Cosmic Ray Small-Scale Anisotropies in Quasi-Linear Theory." In prep.

[15] A. U. Abeysekara et al., Observation of Anisotropy of TeV Cosmic Rays with Two Years of HAWC, Astrophys. J. 865 (2018), no. 1 57, [arXiv: 1805.0184 ].

[16] IceCube, HAWC Collaboration, J. C. D. Velez et al., Combined Analysis of Cosmic-Ray Anisotropy with IceCube and HAWC, PoS ICRC2017 (2018) 539, [arXiv: 1708 .0300]. 